\title{
The use of massive transfusion protocol for trauma and non-trauma patients in a civilian setting: what can be done better?
}

Ramesh Wijaya ${ }^{1}$, MBBS, MRCs, Hui Min Gloria $\underline{\text { Cheng }}^{2}$, MBBS, Chee Keong $\underline{\text { Chong }}{ }^{1}$, MBBS, FRCS

INTRODUCTION Massive transfusion protocol (MTP) is increasingly used in civilian trauma cases to achieve better haemostatic resuscitation in patients requiring massive blood transfusions (MTs), with improved survival outcomes. However, in non-trauma patients, evidence for MTP is lacking. This study aims to assess the outcomes of a newly established MTP in a civilian setting, for both trauma and non-trauma patients, in an acute surgical care unit.

METHODS A retrospective cohort analysis was performed on 46 patients for whom MTP was activated in Changi General Hospital, Singapore. The patients were categorised into trauma and non-trauma groups. Assessment of Blood Consumption (ABC) score was used to identify MTP trauma patients and analyse over-activation rates.

RESULTS Only $39.1 \%$ of all cases with MTP activation eventually received MTs; $39.8 \%$ of the MTs were for non-trauma patients. Mean fresh frozen plasma to packed red blood cells ( $p R B C$ ) ratio achieved with MTP was 0.741 , while mean platelet to pRBC ratio was 0.213 . The 24-hour mortality rate for all patients who received an MT upon MTP activation was $33.3 \%$ (trauma vs. non-trauma group: $45.5 \%$ vs. $14.3 \%$ ). The ABC scoring system used for trauma patients had a sensitivity and specificity of $81.8 \%$ and $41.2 \%$, respectively.

CONCLUSION MTP may be used for both trauma and non-trauma patients in acute care surgery. Scoring systems to predict the need for an MT, improved compliance to predefined transfusion ratios and regular reviews of the MTP are necessary to optimise MTPs and to improve the outcomes of patients receiving MTs.

Keywords: blood components, massive transfusion protocol, mortality, scoring system, trauma

\section{INTRODUCTION}

Massive haemorrhage following trauma is a major cause of death that can be prevented with timely haemostasis and adequate damage control resuscitation. ${ }^{(1)}$ Massive blood transfusions (MTs), defined as the delivery of ten or more units of packed red blood cells in 24 hours, are routinely used to treat uncontrollable haemorrhage. ${ }^{(2)}$

Massive transfusion protocols (MTPs) were first established and developed in the trauma setting in military theatres to facilitate and coordinate large transfusion volumes. These protocols have since been adapted to the civilian trauma setting and involve multilevel work processes within hospitals, thus requiring proper infrastructure, optimal resource allocation and maximal effort. Activation of MTPs, which takes place at the discretion of a senior physician, can be guided by scoring systems that predict the need for MTs. Once activated, MTPs set in motion a string of events to facilitate blood product release, decrease delays and allow timely delivery of blood products from the blood bank to the patient until the protocol ceases. ${ }^{(3,4)}$ MTPs facilitate close adherence to ideal haemostatic resuscitation - a transfusion ratio of 1:1:1 of fresh frozen plasma (FFP), platelets (PLT) and packed red blood cells $(p R B C)^{(5,6)}-$ which helps to improve survival outcomes. Retrospective studies have shown decreased mortality rates in patients receiving increased transfusion ratios of blood products after the establishment of an MTP. ${ }^{(7,8)}$ MTPs, which are now widely used and studied in combat and civilian trauma settings, have been shown to increase the efficiency of transfusion and improve survival outcomes. ${ }^{(9-11)}$

Most studies in the literature focus on the use of MTPs in trauma cases. ${ }^{(12)}$ Studies on the use of MTPs in non-trauma settings are lacking and limited to gastrointestinal tract and major obstetric haemorrhages. ${ }^{(12,13)}$ Thus, the aim of the present study was to assess the efficacy and outcomes of a newly established MTP that was used in an acute surgical care unit for both trauma and non-trauma surgical patients.

\section{METHODS}

Patients for whom MTP was activated in Changi General Hospital (CGH), Singapore, from 1 November 2011 to 30 April 2013 were eligible for inclusion in the present study. Patients who did not receive any blood transfusions and those who were transferred immediately to another centre for further management after MTP activation were excluded. The medical records of the patients who met the inclusion criteria were reviewed and the following data was collected: patient demographics; clinical data at activation and cessation of MTP; amount of blood products transfused; transfusion ratios; and mortality rate. The Assessment of Blood Consumption $(\mathrm{ABC})$ scoring system was used as a predictor for MTs in trauma patients. ${ }^{(14,15)}$ The ABC score is based on four nonweighted parameters: penetrating mechanism; positive Focused Assessment with Sonography for Trauma; systolic blood pressure $\leq 90 \mathrm{mmHg}$ at arrival; and heart rate $\geq 120$ beats per minute (bpm)

${ }^{1}$ Department of General Surgery, Changi General Hospital, ${ }^{2}$ Ministry of Health Holdings Pte Ltd, Singapore

Correspondence: Dr Ramesh Wijaya, Registrar, Department of General Surgery, Changi General Hospital, 2 Simei Street 3, Singapore 529889. ramesh_wijaya@cgh.com.sg 
at arrival. The ABC score for each trauma patient was calculated and data analysis was done using IBM SPSS Statistics version 20.0 (IBM Corp, Armonk, NY, USA).

MTP was implemented in CGH on 1 November 2011. In our institution, the activation of MTPs for patients with uncontrollable bleeding (trauma or non-trauma) requires evaluation by a senior physician. Upon MTP activation, the blood bank sends blood products in three waves: the first wave consists of four units of pRBC, two units of FFP and two units of PLT; the second wave consists of four units of pRBC, FFP and PLT each; and the third wave consists of four units of $\mathrm{pRBC}$, FFP and PLT each, plus an additional ten units of cryoprecipitate (cryoppt), which can be given to the patient at the discretion of the senior physician. In addition to the blood products, adjuncts such as tranexamic acid (TXA) and NovoSeven (N7) may be given to facilitate haemostasis. Intravenous TXA is given to all trauma patients on MTP, while intravenous N7 is given to trauma and non-trauma patients at the discretion of the senior physician. After the first three waves of blood transfusion have been completed, the patient is reassessed by the team to determine whether blood transfusion needs to be continued or terminated.

\section{RESULTS}

Over the 17-month study period (from 1 November 2011 to 30 April 2013), MTP was activated for 50 patients. Among these 50 patients, four patients were excluded from the present study: one did not receive blood transfusion despite MTP activation (i.e. the decision was made to withdraw treatment in view of medical futility) and three patients were transferred to another centre for further management after MTP activation. Thus, a total of 46 patients who had MTP activated and received blood products were included in the present study.

Among these 46 patients, 36 (78.3\%) were male and $10(21.7 \%)$ were female. The mean age of the patients was $55.67 \pm 19.36$ years. Most of the patients $(n=28,60.9 \%)$ had MTP activated for trauma, while 18 (39.1\%) had MTP activated for non-traumatic events such as bleeding gastrointestinal tract, ruptured abdominal aortic aneurysm, ruptured splenic artery aneurysm and intraoperative or postoperative bleeding (Table I).

Only 18 of the 46 patients $(39.1 \%$ ) received MTs (i.e. $\geq 10$ units of pRBC every 24 hours $)-11(61.1 \%)$ were trauma patients and 7 (38.9\%) were non-trauma patients. The mean MT FFP:pRBC ratio and mean PLT:pRBC ratio of all patients who received MTs, including the trauma and non-trauma patients, are shown in Table II.

The overall and 24-hour mortality rates of all the patients who received MTs are shown in Table III. The results showed that for the patients who received MTs on MTP, $75.0 \%$ of the deaths occurred within the first 24 hours. For trauma and nontrauma patients who received MTs on MTP, $83.3 \%$ and $50.0 \%$ of the deaths, respectively, occurred within the first 24 hours. Among the 28 patients who did not receive MTs, 5 (17.9\%) died within 24 hours; the mean mortality time of these five patients was $4.40 \pm 3.36$ hours.
Table I. Diagnosis of the non-trauma patients $(n=18)$.

\begin{tabular}{lc}
\hline Diagnosis & No. (\%) \\
\hline Bleeding gastrointestinal tract & $12(66.7)$ \\
Ruptured abdominal aortic aneurysm & $3(16.7)$ \\
Ruptured splenic artery aneurysm & $1(5.6)$ \\
Intraoperative bleeding & $1(5.6)$ \\
Postoperative bleeding & $1(5.6)$ \\
\hline
\end{tabular}

Table II. Mean ratios of the blood products transfused in the patients who received massive blood transfusion (MT).

\begin{tabular}{lcc}
\hline Patient group & \multicolumn{2}{c}{ Mean \pm SD ratio } \\
\cline { 2 - 3 } & FFP:pRBC & PLT:pRBC \\
\hline $\begin{array}{l}\text { All patients who received } \\
\text { MTs }(\mathbf{n}=\mathbf{1 8})\end{array}$ & $0.741 \pm 0.238$ & $0.213 \pm 0.145$ \\
$\quad$ Trauma patients $(n=11)$ & $0.796 \pm 0.257$ & $0.259 \pm 0.163$ \\
$\quad$ Non-trauma patients $(n=7)$ & $0.655 \pm 0.192$ & $0.141 \pm 0.072$ \\
\hline
\end{tabular}

FFP: fresh frozen plasma; PLT: platelets; pRBC: packed red blood cells; SD: standard deviation

Table III. Overall mortality and 24-hour mortality of the patients who received massive blood transfusions.

\begin{tabular}{lccc}
\hline Variable & \multicolumn{3}{c}{ No. (\%) } \\
\cline { 2 - 4 } & $\begin{array}{c}\text { All } \\
\text { patients } \\
(\mathbf{n}=\mathbf{1 8})\end{array}$ & $\begin{array}{c}\text { Trauma } \\
\text { patients } \\
(\mathbf{n}=\mathbf{1 1 )}\end{array}$ & $\begin{array}{c}\text { Non-trauma } \\
\text { patients } \\
(\mathbf{n}=\mathbf{7})\end{array}$ \\
\hline Overall mortality & $8(44.4)$ & $6(54.5)$ & $2(28.6)$ \\
24-hour mortality & $6(33.3)$ & $5(45.5)$ & $1(14.3)$ \\
\hline
\end{tabular}

TXA was administered to 15 of the 18 patients (83.3\%) who received MTs. As predetermined in the MTP guidelines, all 11 trauma patients who received MTs also received TXA. TXA was given to $4(57.1 \%)$ of the seven non-trauma patients who received MT at the attending physician's discretion. Among the 18 patients who received MTs, N7 was given to 8 (44.4\%) patients and cryoppt was given to $12(66.7 \%)$ patients, at the attending physicians' discretion. The 24-hour mortality rates of the 18 patients who received MTs were as follows: (a) 40.0\% for patients who received both MTs and TXA, and $0 \%$ for those who received MTs but not TXA; (b) $12.5 \%$ for patients who received both MTs and N7, and 50.0\% for those who received MTs but not N7; (c) $41.7 \%$ for patients who received both MTs and cryoppt, and $16.7 \%$ for those who received MTs but not cryoppt.

Among the 28 trauma patients in our study cohort $(n=46)$, 19 (67.9\%) patients had an $A B C$ score $\geq 2$. When an $A B C$ score $\geq 2$ was used as the criteria for MTP activation, we found that it had a sensitivity of $81.8 \%$ and specificity of $41.2 \%$ for predicting the need for MTs in our study cohort of trauma patients.

\section{DISCUSSION}

There are concerns about inefficient resource allocation when MTP is activated for patients who ultimately do not require MTs. ${ }^{(16)}$ This is defined as over-activation, which can be quantified as the percentage of patients who ultimately did not receive MTs even though MTP was activated. In the present study, only $39.1 \%$ of 
all the patients who had MTP activated eventually required MTs (i.e. the over-activation rate was $60.9 \%$ ). Although there was no significant difference between the over-activation rates of the trauma and non-trauma groups $(60.7 \%$ and $61.1 \%$, respectively), the over-activation rate of the non-trauma group was closer to the rates reported in the literature $53.8 \%$ and $51 \%$ for similar groups of non-trauma patients in studies by McDaniel et al and Morse et al, respectively). ${ }^{(16,17)}$ The MTP over-activation rates reported for trauma patients in the literature are considerably lower than that found in the present study - McDaniel et al ${ }^{(17)}$ reported a $19.2 \%$ trauma over-activation rate, while Morse et al ${ }^{(16)}$ reported a $29 \%$ trauma over-activation rate.

In the present study, we attempted to use a validated algorithm scoring system to predict MT requirement so as to reduce overactivation. However, the use of the $\mathrm{ABC}$ scoring system as a prerequisite for MTP activation had a sensitivity of $81.8 \%$ and specificity of $41.2 \%$ when an $A B C$ score $\geq 2$ was used as the cutoff point to predict MT requirement. This differs from the findings of Nunez et al's study, which reported a sensitivity and specificity of $75 \%$ and $86 \%$, respectively. ${ }^{(14)}$ In other words, the ABC scoring system would not have helped reduce the over-activation rate in our study cohort. Although the ABC scoring system is useful for predicting patients who need MTs with MTP in other populations, there is a need to identify scoring systems that are more suitable for use in our patient population. ${ }^{(18,19)}$ Currently, there are no validated scoring systems for predicting MT requirement in nontrauma patients. In a civilian setting where non-trauma patients form a considerable portion of patients requiring MTs with MTP (as was the case in the present study), it may be timely to consider creating scoring systems targeted at this group of patients.

In trauma cases, the first 24 hours represent the most lethal period. ${ }^{(20)}$ In the present study, $83.3 \%$ of the deaths of trauma patients who received MTs on MTP occurred within the first 24 hours. We also observed that the 24-hour mortality rate of the trauma patients was higher than that of the non-trauma patients (i.e. $45.5 \%$ and $14.3 \%$, respectively). This finding is supported by the study by Morse et al, in which the 24-hour mortality rates of the trauma and non-trauma patients were $59 \%$ and $35 \%$, respectively. ${ }^{(16)}$ The poorer initial survival outcome of trauma patients receiving MTs on MTP may be due to acute coagulopathy caused by trauma.

A study by Sperry et al, which examined the factors associated with improved early trauma survival outcomes, found that a high FFP:pRBC ratio (i.e. $\geq 1: 1.5$ ) lowered the 24-hour mortality rate from $12.8 \%$ to $3.9 \% .{ }^{(21)}$ The MTP used in the present study, which had a FFP:PLT:pRBC target ratio of $1: 1: 1$, achieved a mean FFP:pRBC ratio of 0.741 and a mean PLT:pRBC ratio of 0.213 . Deviation from the 1:1:1 target ratio was also observed in Shaz et al's study involving 132 civilian trauma patients; in that study, the MTP achieved a mean FFP:pRBC ratio of 0.5 and a mean PLT:pRBC ratio of 0.6. ${ }^{(22,23)}$ Evidently, while an ideal transfusion ratio of 1:1:1 may be proposed in MTPs, it has been suggested that this target is not always achievable immediately. Notably, studies have shown that failure to achieve these ratios is associated with higher mortality. ${ }^{(22,24)}$ Cotton et al found that patients whose MT activations complied with the predefined FFP:PLT:pRBC ratio achieved lower 24-hour mortality rates than those whose MT activations did not comply with the predefined ratio. ${ }^{(24)}$ Hence, constant review, optimised resource activation and allocation, and strict compliance to the MTP are necessary to ensure that the transfusion ratios of blood products are closer to the ideal ratio.

Recently, the use of alternative non-surgical haemostatic adjuncts in MTP (such as TXA, N7, prothrombin and fibrinogen concentrates) to deal with the complications of trauma- and MT-induced coagulopathy has been investigated and discussed substantially. However, the role of these adjuncts in MTP (apart from TXA, which is recommended for use in the early resuscitative process), is still largely unclear. ${ }^{(25,26)}$ Although some randomised controlled trials investigating the use of N7 have shown improved outcomes in trauma patients who received MTs and a positive effect in non-traumatic causes of haemorrhage, more recent studies have failed to show any significant benefits. ${ }^{(27-30)}$ As such, evidence on the role of N7 in MTP is still conflicting and debatable. In the present study, patients who received MTs and N7 had a lower 24-hour mortality rate than the patients who received MTs but not N7 (16.7\% vs. 58.3\%). Although our data suggests that N7 improves mortality outcomes in MTs, the presence of selection and survival bias with regard to the use of haemostatic adjuncts (i.e. heavy reliance on physician-directed use of the agents, rather than the use of objective indicators for usage of these agents) cannot be ignored.

Similarly, based on our results, we were unable to conclusively establish an association between the use of cryoppt and survival. The results from studies that examined the use of cryoppt for massive haemorrhage have been generally inconclusive. The recent multicentre PROMMT (PRospective, Observational, Multicenter, Major trauma Transfusion) study did not identify any association between the use of cryoppt and in-hospital mortality. ${ }^{(31)}$ However, the authors of that study have acknowledged that there are significant variations in the use of cryoppt among centres and have encouraged randomised controlled studies to be conducted to achieve a more accurate conclusion. ${ }^{(31)}$ With such scant and conflicting evidence on the use of haemostatic adjuncts, even in settings not involving MTPs, the role of such agents (especially N7) in MTP is thus limited. As our results are descriptive in nature, there is a need for further investigation on the use of such agents in MTP, ideally in a trial setting.

The overall mortality rate of our study cohort (in both the trauma and non-trauma groups) who received MTs was $44.4 \%$. Sinha et al's study of MTP use in a similar civilian setting reported a $29 \%$ overall mortality rate among their study cohort of trauma and non-trauma patients. ${ }^{(32)}$ The difference in overall mortality rates between Sinha et al's study and our study could be due to the precocious analysis of our centre's MTP at 17 months. As MTP usage matures with time, compliance is likely to improve, resulting in improved mortality outcomes. ${ }^{(33)}$

The present study was limited by its retrospective study design and small sample size. There was also survival bias, as $17.9 \%$ of the patients who did not receive MT died within 
24 hours (mean mortality time of 4.40 hours). If these patients had survived beyond 24 hours, they might have met the criteria for receiving an MT. Nonetheless, the present study highlighted key aspects and considerations in the implementation of MTP in the management of MTs in both trauma and non-trauma acute surgical care patients, in spite of the unpredictability that exists in haemorrhagic emergencies, especially following trauma.

Although MTPs are widely used and studied in combat and civilian trauma settings to improve the survival rates of patients with massive haemorrhage, they are less frequently used in non-trauma patients. ${ }^{(34)}$ In the present study, we found that nontrauma patients formed a considerable proportion of all patients (38.9\%) who ultimately received MTs after MTP activation. Thus, the use of MTP could be extended to non-trauma patients in an acute surgical unit, to improve survival outcomes for this group of patients. Attention should be given to over-activation rates, compliance to transfusion ratios and mortality, as well as the differences between trauma and non-trauma patients to ensure optimal use of MTP. Well-designed prospective studies to evaluate MTP performance are warranted, especially in the non-trauma setting.

To conclude, the establishment of MTPs to achieve better survival outcomes in patients requiring MTs may be beneficial for both trauma and non-trauma patients. The results of the present study suggest that the efficiency of MTP delivery can be improved by optimising the activation rate, evaluating the compliance to ideal transfusion ratios, and by identifying differences between trauma and non-trauma populations receiving MTs. Meticulous evaluation is required to further refine MTPs so that optimal outcomes for patients can be achieved.

\section{REFERENCES}

1. Teixeira PG, Inaba K, Hadjizacharia P, et al. Preventable or potentially preventable mortality at a mature trauma center. J Trauma 2007; 63:133846; discussion 1346-7.

2. British Committee for Standards in Haematology, Stainsby D, MacLennan $\mathrm{S}$, et al. Guidelines on the management of massive blood loss. Br J Haematol 2006; 135:634-41.

3. Greer SE, Rhynhart KK, Gupta R, Corwin HL. New developments in massive transfusion in trauma. Curr Opin Anaesthesiol 2010; 23:246-50.

4. Griffee MJ, Deloughery TG, Thorborg PA. Coagulation management in massive bleeding. Curr Opin Anaesthesiol 2010; 23:263-8.

5. Holcomb JB, Jenkins D, Rhee $P$, et al. Damage control resuscitation: directly addressing the early coagulopathy of trauma. J Trauma 2007; 62:307-10.

6. Johnson JL, Moore EE, Kashuk JL, et al. Effect of blood products transfusion on the development of postinjury multiple organ failure. Arch Surg 2010; 145:973-7.

7. Dente CJ, Shaz BH, Nicholas JM, et al. Improvements in early mortality and coagulopathy are sustained better in patients with blunt trauma after institution of a massive transfusion protocol in a civilian level I trauma center. J Trauma 2009; 66:1616-24.

8. Johansson PI, Stensballe J. Effect of Haemostatic Control Resuscitation on mortality in massively bleeding patients: a before and after study. Vox Sang 2009; 96:111-8.

9. Malone DL, Hess JR, Fingerhut A. Massive transfusion practices around the globe and a suggestion for a common massive transfusion protocol. J Trauma 2006; 60(6 Suppl):S91-6.

10. Cap AP, Spinella PC, Borgman MA, Blackbourne LH, Perkins JG. Timing and location of blood product transfusion and outcomes in massively transfused combat casualties. J Trauma Acute Care Surg 2012; 73(2 Suppl 1):S89-94.

11. Cotton BA, Au BK, Nunez TC, et al. Predefined massive transfusion protocols are associated with a reduction in organ failure and postinjury complications. J Trauma 2009; 66:41-8; discussion 48-9.

12. Ciccocioppo A, Walker M, Taylor F, Padbury R, Wattchow D. Protocol management for patients presenting with lower Gl haemorrhage saves costs and maintains outcomes. ANZ J Surg 2011; 81:451-5.

13. Onwuemene O, Green D, Keith L. Postpartum hemorrhage management in 2012: predicting the future. Int J Gynaecol Obstet 2012; 119:3-5.

14. Nunez TC, Voskresensky IV, Dossett LA, et al. Early prediction of massive transfusion in trauma: simple as ABC (assessment of blood consumption)? J Trauma 2009; 66:346-52.

15. Cotton BA, Dossett LA, Haut ER, et al. Multicenter validation of a simplified score to predict massive transfusion in trauma. J Trauma 2010; 69 Suppl 1:S33-9.

16. Morse BC, Dente CJ, Hodgman El, et al. Outcomes after massive transfusion in nontrauma patients in the era of damage control resuscitation. Am Surg 2012; 78:679-84.

17. McDaniel LM, Neal MD, Sperry JL, et al. Use of a massive transfusion protocol in nontrauma patients: activate away. J Am Coll Surg 2013; 216:1103-9.

18. McLaughlin DF, Niles SE, Salinas J, et al. A predictive model for massive transfusion in combat casualty patients. J Trauma 2008; 64(2 Suppl):S57-63; discussion S63.

19. Schreiber MA, Perkins J, Kiraly L, et al. Early predictors of massive transfusion in combat casualties. J Am Coll Surg 2007; 205:541-5.

20. Acosta JA, Yang JC, Winchell RJ, et al. Lethal injuries and time to death in a level I trauma center. J Am Coll Surg 1998; 186:528-33.

21. Sperry JL, Ochoa JB, Gunn SR, et al; Inflammation the Host Response to Injury Investigators. An FFP: PRBC transfusion ratio $>/=1: 1.5$ is associated with a lower risk of mortality after massive transfusion. J Trauma 2008; 65:986-93.

22. Shaz BH, Dente CJ, Nicholas J, et al. Increased number of coagulation products in relationship to red blood cell products transfused improves mortality in trauma patients. Transfusion 2010; 50:493-500.

23. Holcomb JB, Wade CE, Michalek JE, et al. Increased plasma and platelet to red blood cell ratios improves outcome in 466 massively transfused civilian trauma patients. Ann Surg 2008; 248:447-58.

24. Cotton BA, Dossett LA, Au BK, et al. Room for (performance) improvement: provider-related factors associated with poor outcomes in massive transfusion. J Trauma 2009; 67:1004-12.

25. Pham HP, Shaz BH. Update on massive transfusion. Br J Anaesth 2013; 111 Suppl 1:i71-82.

26. CRASH-2 trial collaborators, Shakur H, Roberts I, et al. Effects of tranexamic acid on death, vascular occlusive events, and blood transfusion in trauma patients with significant haemorrhage (CRASH-2): a randomised, placebocontrolled trial. Lancet 2010; 376:23-32.

27. Boffard KD, Riou B, Warren B, et al; NovoSeven Trauma Study Group. Recombinant factor VIla as adjunctive therapy for bleeding control in severely injured trauma patients: two parallel randomized, placebocontrolled, double-blind clinical trials. J Trauma 2005; 59:8-15; discussion $15-8$.

28. Tsai TC, Rosing JH, Norton JA. Role of factor VII in correcting dilutional coagulopathy and reducing re-operations for bleeding following nontraumatic major gastrointestinal and abdominal surgery. J Gastrointest Surg 2010; 14:1311-8.

29. Hauser CJ, Boffard K, Dutton R, et al; CONTROL Study Group. Results of the CONTROL trial: efficacy and safety of recombinant activated Factor VII in the management of refractory traumatic hemorrhage. J Trauma 2010; 69:489-500.

30. Simpson E, Lin Y, Stanworth S, et al. Recombinant factor VIla for the prevention and treatment of bleeding patients without haemophilia. Cochrane Database Syst Rev 2012; 3:CD005011.

31. Holcomb JB, Fox EE, Zhang X, et al; PROMMTT Study Group. Cryoprecipitate use in the PROMMTT study. J Trauma Acute Care Surg 2013; 75(1 Suppl 1):S31-9.

32. Sinha R, Roxby D, Bersten A. Experience with a massive transfusion protocol in the management of massive haemorrhage. Transfus Med 2013; 23:108-13.

33. Nunez TC, Young PP, Holcomb JB, Cotton BA. Creation, implementation, and maturation of a massive transfusion protocol for the exsanguinating trauma patient. J Trauma 2010; 68:1498-505.

34. Neal MD, Marsh A, Marino R, et al. Massive transfusion: an evidencebased review of recent developments. Arch Surg 2012; 147: 563-71. 